\title{
Treatment of $\gamma$-Hydroxybutyric Acid and $\gamma$-Butyrolactone Overdose with Two Potent Monocarboxylate Transporter 1 Inhibitors, AZD3965 and AR-C155858
}

\author{
Kristin E. Follman and (D) Marilyn E. Morris \\ Department of Pharmaceutical Sciences, School of Pharmacy and Pharmaceutical Sciences, University at Buffalo, State \\ University of New York, Buffalo, New York
}

Received January 14, 2019; accepted April 17, 2019

\begin{abstract}
The illicit use of $\gamma$-hydroxybutyric acid (GHB), and its prodrug, $\gamma$-butyrolactone (GBL), results in severe adverse effects including sedation, coma, respiratory depression, and death. Current treatment of GHB/GBL overdose is limited to supportive care. Recent reports indicate that GHB-related deaths are on the rise; a specific treatment may reduce lethality associated with GHB/GBL. Pretreatment with inhibitors of monocarboxylate transporter 1 (MCT1), a transporter that mediates many of the processes involved in the absorption, distribution (including brain uptake), and elimination of GHB/GBL, has been shown to prevent GHB-induced respiratory depression by increasing the renal clearance of GHB. To identify whether MCT1 inhibition is an effective treatment of GHB overdose, the impact of two MCT1 inhibitors, (S)-5-(4-hydroxy-4-methylisoxazolidine-2-carbonyl)1-isopropyl-3-methyl-6-((3-methyl-5-(trifluoromethyl)-1H-pyrazol4-yl)methyl)thieno[2,3-day]pyrimidine-2,4(1H,3H)-dione (AZD3965) and 6-[(3,5-dimethyl-1H-pyrazol-4-yl)methyl]-5-[((4S)-4-hydroxy-2-
\end{abstract}

isoxazolidinyl]carbonyl]-3-methyl-1-(2-methylpropyl)thieno[2,3day]pyrimidine2,4(1H,3H)-dione (AR-C155858), on the toxicokinetics and toxicodynamics of GHB/GBL was assessed when the administration of the inhibitor was delayed 60 and 120 minutes (post-treatment) after administration of GHB/GBL. AR-C155858 and AZD3965 reduced the toxicodynamic effects of GHB when GHB was administered intravenously, orally, or orally as the prodrug GBL. The impact of these inhibitors on GHB toxicokinetics was dependent on the route of GHB administration and the delay between GHB/GBL administration and administration of the MCT1 inhibitor. The reduction in GHB plasma exposure did not explain the observed effect of MCT1 inhibition on GHB-induced respiratory depression. The efficacy of MCT1 inhibition on GHB toxicodynamics is likely driven by the pronounced reduction in GHB brain concentrations. Overall, this study indicates that inhibition of MCT1 is an effective treatment of GHB/GBL overdose.

\section{Introduction}

$\gamma$-Hydroxybutyric acid (GHB) is an endogenous short chain fatty acid that is present in the central nervous system of humans (White, 2017). GHB was synthesized as a structural analog of GABA, the major inhibitory neurotransmitter in the brain (White, 2017). GHB has several pharmacological uses, including the treatment of narcolepsy (available in the United States, Canada, and Europe as Xyrem) and alcohol withdrawal (available in Austria and Italy as Alcover), and is used as an anesthetic (availablein Germany as Somsanit) (Carter et al., 2009). The utility of the compound is severely limited by its potential for illicit use. GHB is abused for several of its desirable effects including euphoria, decreased inhibition, and enhancement of growth hormone release (White, 2017). When

This work was supported by the National Institutes of Health National Institute on Drug Abuse [Grant R01 DA023223].

https://doi.org/10.1124/jpet.119.256503.

S This article has supplemental material available at jpet.aspetjournals.org.
GHB is abused, it has many harmful and potentially fatal side effects including hypothermia, coma, and respiratory depression (White, 2017). GHB is currently a schedule $1 / 3$ controlled substance, while $\gamma$-butyrolactone (GBL), a prodrug of GHB, is more readily available as a list 1 controlled substance (Goodwin et al., 2009; White, 2017). GBL is rapidly absorbed and converted to GHB, with the resulting GHB responsible for the toxicodynamics (TD) effects observed with GBL (Giarman and Roth, 1964; Lettieri and Fung, 1976; Goodwin et al., 2009). Recently, an increase in GHB abuse has been recorded. Over 20 GHB-associated deaths were reported in 2015 alone in a London-based study (Hockenhull et al., 2017). This was a $119 \%$ increase in GHB-associated deaths compared with the previous year (Hockenhull et al., 2017). Currently, there is no specific treatment for GHB/GBL overdose, and treatment is limited to supportive care for overdosed individuals.

GHB exhibits nonlinear kinetics effects that are mediated by several saturable processes including absorption, metabolism, and renal reabsorption (Morris et al., 2005; Vijay et al.,

ABBREVIATIONS: ABEC, area below the effect curve; AR-C155858, 6-[(3,5-dimethyl-1H-pyrazol-4-yl)methyl]-5-[[(4S)-4-hydroxy-2isoxazolidinyl]carbonyl]-3-methyl-1-(2-methylpropyl)thieno[2,3-day]pyrimidine2,4(1H,3H)-dione; AUC, area under the curve; AZD3965, (S)-5(4-hydroxy-4-methylisoxazolidine-2-carbonyl)-1-isopropyl-3-methyl-6-((3-methyl-5-(trifluoromethyl)-1H-pyrazol-4-yl)methyl)thieno[2,3-day] pyrimidine-2,4(1H,3H)-dione; BBB, blood-brain barrier; CL/F, oral clearance; $\mathrm{CL}_{\mathrm{R}}$, renal clearance; GBL, $\gamma$-butyrolactone; GHB, $\gamma$-hydroxybutyric acid; GHB-d6, deuterated $\gamma$-hydroxybutyric acid; MCT1, monocarboxylate transporter $1 ; t_{\mathrm{d}}$, duration of effect; TD, toxicodynamics; TK, toxicokinetics. 
2015). GHB has a $\mathrm{p} K_{\mathrm{a}}$ value of 4.7 , which results in the compound being ionized at physiologic $\mathrm{pH}$ (Marinetti et al., 2005). Due to this ionization, GHB is unable to pass freely through cell membranes, making transport via membrane proteins an important factor in GHB toxicokinetics (TK). GHB is a substrate of monocarboxylate transporter 1 [MCT1 (SLC16A1)], a protondependent transporter that exhibits ubiquitous tissue distribution in humans (Morris and Felmlee, 2008). The TD effects of GHB, including respiratory depression, have been shown to be mediated by GHB agonism of $\mathrm{GABA}_{\mathrm{B}}$ receptors in the brain, making this organ the site of action for GHB (Carai et al., 2001; Jensen and Mody, 2001; Kaupmann et al., 2003; Goodwin et al., 2005; Morse et al., 2012; Morse and Morris, 2013b). MCT1 is the only monocarboxylate transporter expressed at the blood-brain barrier (BBB) and mediates the uptake of GHB into the brain (Vijay and Morris, 2014). MCT1 is also present in the kidney, and mediates the facilitated reabsorption of GHB that has been filtered from the blood, and in this way GHB renal clearance $\left(\mathrm{CL}_{\mathrm{R}}\right)$ is limited by MCT1 (Morris et al., 2005). Based on the role of MCT1 in GHB brain uptake and GHB renal reabsorption, MCT1 is a potential target for GHB overdose treatment. GHB is also a substrate for the sodium-dependent MCT1; this transporter may play an important role in the intestine and kidney. Further studies are needed to fully characterize the contribution of this transporter to GHB TK.

It has been demonstrated previously, both preclinically and clinically, that MCT1 inhibition can increase the $\mathrm{CL}_{R}$ value, resulting in an increase in the total clearance for GHB (Morris et al., 2005, 2011; Morse and Morris, 2013b; Vijay et al., 2015). MCT1 inhibition also has the potential to limit the brain exposure, and therefore the TD effects of GHB. The effect of (S)-5-(4-hydroxy-4-methylisoxazolidine-2-carbonyl)-1-isopropyl3-methyl-6-((3-methyl-5-(trifluoromethyl)-1H-pyrazol-4-yl)methyl)thieno[2,3-day]pyrimidine-2,4(1H,3H)-dione (AZD3965) and 6-[(3,5-dimethyl-1H-pyrazol-4-yl)methyl]-5-[[(4S)-4hydroxy-2-isoxazolidinyl]carbonyl]-3-methyl-1-(2-methylpropyl) thieno[2,3-day]pyrimidine2,4(1H,3H)-dione (AR-C155858) on GHB TK and TD were investigated with GHB administered intravenously and GHB/GBL administered orally, which is the typical route of administration for abuse. The aim of this study was to investigate the utility of two potent MCT1 inhibitors, AZD3965 and AR-C155858, for the treatment of GHB/GBL overdose.

\section{Materials and Methods}

Chemical and Reagents. Sodium GHB was provided by the National Institute on Drug Abuse. GBL was purchased from Sigma (St. Louis, MO). AZD3965 was provided by MedKoo (Morrisville, NC). AR-C155858 was purchased from Chemscene (Monmouth Junction, NJ). Deuterated GHB (GHB-d6) was purchased from Cerilliant Corporation (Round Rock, TX).

Animals and Surgery. Male Sprague-Dawley rats (Envigo, Somerset, NJ) were housed under controlled temperature and humidity with an artificial 12-hour light/dark cycle and food was available ad libitum. Jugular vein cannulae were surgically implanted under anesthesia with a mixture of ketamine/xylazine. Cannulae were flushed daily with $40 \mathrm{IU} / \mathrm{ml}$ heparinized saline to maintain patency. Rats were allowed a minimum of 72 hours for recovery from surgery before the experiments were conducted. Animals used for experiments weighed between 230 and $330 \mathrm{~g}$ on the day of the experiment. All animal procedures were approved by the University at Buffalo Institutional Animal Care and Use Committee.
Toxicokinetic/Toxicodynamic Studies. The impact of MCT1 inhibition with AZD3965 and AR-C155858 on the toxicokinetics and toxicodynamics was assessed using whole-body plethysmography and serial blood and urine sampling (model PLY4213; Buxco Research Systems, Wilmington, NC). Studies consisted of administration of intravenous or oral GHB and oral GBL, followed by the administration of intravenous AZD3965 or AR-C155858. GHB and GBL were administered as a $300 \mathrm{mg} / \mathrm{ml}$ solution in sterile, double-distilled water. AZD3965 and AR-C155858 were administered as $1 \mathrm{mg} / \mathrm{ml}$ solutions in sterile normal saline with $20 \%$ cyclodextrin (w/v). For studies including the oral administration of GHB/GBL, animals were fasted 12-15 hours prior to the administration of GHB/GBL. On the day of the study jugular-cannulated rats were placed in plethysmography chambers and allowed to acclimate for 45 minutes prior to the recording of five baseline measurements of respiration over $15 \mathrm{~min}$ utes. After baseline measurements were obtained, GHB or GBL was administered either intravenously via the jugular cannula or orally via oral gavage.

The time of administration of GHB/GBL was considered time 0 . Treatment compounds (AZD3965 or AR-C155858) were administered either 5 minutes prior to GHB or 60 or 120 minutes after GHB/GBL. Respiration measurements were recorded at 2.5, 5, 7.5, 10, 15, 20, 25, and 30 minutes and every 15 minutes thereafter until the end of the study. Additional respiration measurements were taken 5 and 10 minutes after the administration of the treatment compound, either at 65 and 70 minutes or 125 and 130 minutes.

For intravenous GHB (14.4 mmol/kg) and oral GBL $(5.77 \mathrm{mmol} / \mathrm{kg})$, the duration of the study was 8 hours, and blood samples were taken at $3,11,21,31,61,121,181,241,301,331,361$, and 481 minutes; these samples were taken immediately following the corresponding respiration measurement. Urine was collected in intervals from 0 to 1,1 to 2, 2 to 4,4 to 6 , and 6 to 8 hours. For oral GHB (14.4 mmol $/ \mathrm{kg})$, the study duration was 15 hours, and blood samples were taken at 3,15 , $31,61,121,181,241,361,481,601,721$, and 921 minutes. Urine was collected in intervals from 0 to 2,2 to 4,4 to 6,6 to 12 , and 12 to 15 hours.

Brain-to-Plasma Partitioning of GHB after Oral GHB Administration. Brain-to-plasma partitioning was assessed with oral GHB administration with and without treatment with AZD3965. Male Sprague-Dawley rats were administered $14.4 \mathrm{mmol} / \mathrm{kg}$ GHB via oral gavage after fasting for 12-15 hours. Control groups (without AZD3965 treatment) were sacrificed at 65 and 120 minutes after GHB administration. Treatment groups were administered $5 \mathrm{mg} / \mathrm{kg}$ AZD3965 intravenously via jugular vein cannulae 60 minutes after GHB administration. Treatment groups were sacrificed at 65 and 120 minutes after GHB administration, corresponding to 5 and 60 minutes after AZD3965 administration. Terminal plasma and whole brain samples were collected. Brain samples were snap-frozen in liquid nitrogen, and brain and plasma samples were stored at $-80^{\circ} \mathrm{C}$ until analysis. Control and treatment groups consisted of four animals each.

Sample Analysis. Plasma and urine samples were analyzed for GHB utilizing a previously published liquid chromatography/mass spectrometry assay (Felmlee et al., 2010; Morse et al., 2012). In brief, plasma samples were prepared by adding $5 \mu \mathrm{l}$ of sample plasma to $50 \mu \mathrm{l}$ of blank plasma (for samples with lower GHB concentrations, $50 \mu \mathrm{l}$ of sample plasma was used without the addition of blank plasma). For GHB standards, $5 \mu$ l of standard stock solution was added to $50 \mu$ l blank plasma. The internal standard, GHB-d6, was added to all samples $(5 \mu \mathrm{l})$. Proteins were precipitated with the addition of $800 \mu \mathrm{l}$ acetonitrile, and samples were centrifuged for 20 minutes at $10,000 \mathrm{rpm}$ at $4^{\circ} \mathrm{C}$. An aliquot of the remaining supernatant $(750 \mu \mathrm{l})$ was dried under a stream of nitrogen and reconstituted in $250 \mu \mathrm{l}$ of aqueous mobile phase. Urine samples were prepared by diluting urine samples $(100 \times)$ and adding $5 \mu$ l of GHB-d6. Standards were prepared by adding $5 \mu \mathrm{l}$ of GHB-d6 and $5 \mu \mathrm{l}$ of standard stock solution to $25 \mu \mathrm{l}$ dilute blank urine. Double-distilled water ( $465 \mu \mathrm{l}$ for standards; 470 for samples) and $1 \mathrm{ml}$ methanol were then added. Samples were centrifuged for 20 minutes at $10,000 \mathrm{rpm}$ at 


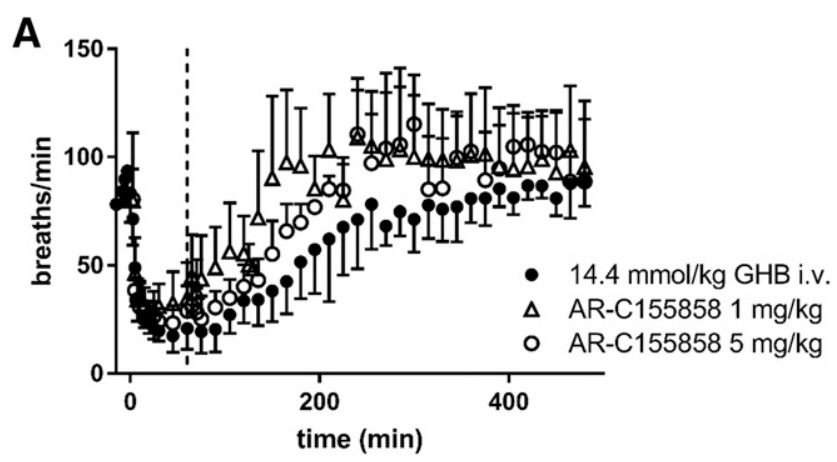

B

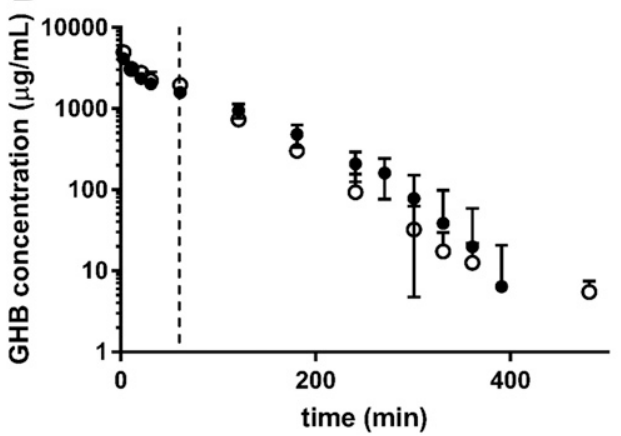

Fig. 1. Effect of AR-C155858 on intravenous GHB-induced respiratory depression (A) and GHB toxicokinetics (B). GHB was administered as a $14.4 \mathrm{mmol} / \mathrm{kg}$ intravenous bolus at time 0 . AR-C155858 was administered as an intravenous bolus 60 minutes later, at a dose of $1 \mathrm{mg} / \mathrm{kg}$ (open triangles) or $5 \mathrm{mg} / \mathrm{kg}$ (open circles). Data are presented as mean \pm S.D. Time of AR-C155858 administration is indicated as a dashed line. Control data (closed circles) for breathing frequency were obtained from Morse and Morris (2013a); control data for GHB TK were obtained from Vijay et al. (2015). $n=3-7$ ( $n=2$ for panel B, AR-C155858 treatment group).

$4^{\circ} \mathrm{C}$ and the supernatant was transferred to a vial for analysis. Due to the rapid conversion of GBL to GHB, in vivo GBL concentrations were not assessed. For both the administration of GHB and GBL, GHB plasma and urine concentrations were evaluated for toxicokinetics.

Brain samples were also analyzed as described previously (Felmlee et al., 2010). Whole brain samples were homogenized in $4 \mathrm{ml} / \mathrm{g}$ doubledistilled water. For samples, $10 \mu \mathrm{l}$ of GHB-d6 was added to $100 \mu \mathrm{l}$ of brain homogenate. For standards, $10 \mu \mathrm{l}$ of standard stock solution and GHB-d6 were added to $100 \mu \mathrm{l}$ of blank brain homogenate. Doubledistilled water ( $880 \mu \mathrm{l}$ for standards; $890 \mu \mathrm{l}$ for samples) and $1 \mathrm{ml}$ acetonitrile were added to samples, which were then centrifuged for 20 minutes at $10,000 \mathrm{rpm}$ at $4^{\circ} \mathrm{C}$. Supernatant was transferred to a vial for analysis.

Data/Statistical Analysis. Control data for breathing frequency were obtained from Morse and Morris (2013a), and control data for GHB TK were obtained from Vijay et al. (2015). Toxicokinetic and toxicodynamic data were plotted in GraphPad Prism 7, GraphPad Software. The main toxicodynamic parameter of interest was the area below the effect curve (ABEC) for breathing frequency, where the
ABEC was identified using the individual baseline for each animal, ignoring peaks that were less than $10 \%$ of the distance from the minimum to the maximum $y$-value and peaks that contained less than two adjacent points. The first negative peak area was used for the ABEC in this analysis. The duration of effect $\left(t_{\mathrm{d}}\right)$ was defined as the length of time for the first negative peak. The maximal effect was the minimum value for breathing frequency observed.

Toxicokinetic data were analyzed using the PK solver add-in for Microsoft Excel (Zhang et al., 2010). Toxicokinetic parameters of interest were the area under the curve (AUC), total clearance (for intravenous data), oral clearance (CL/F) over bioavailability (for oral data), renal clearance, nonrenal clearance, and urinary recovery (presented as the percentage of administered dose). In addition, the $C_{\max }$ and time of maximum concentration were determined for oral data. The AUC was determined using the linear trapezoidal method, clearance was dose/AUC, and $\mathrm{CL}_{\mathrm{R}}$ was determined as the amount of GHB excreted unchanged in urine/AUC. Nonrenal clearance was defined as the difference between clearance and $\mathrm{CL}_{R}$.

Statistical significance was determined using one-way ANOVA with Dunnett's post hoc test for comparisons of multiple treatment groups to a control. For comparisons of a single group to a control, a Student's $t$ test was used. All statistics were performed in GraphPad Prism 7.

\section{Results}

Impact of AR-C155858 on Intravenous GHB TK/TD. The administration of AR-C155858 60 minutes after intravenous GHB resulted in an increase in breathing frequency (Fig. 1A). The reduction in ABEC, maximal effect, and $t_{\mathrm{d}}$ was statistically significant for $1 \mathrm{mg} / \mathrm{kg}$ AR-C155858, but not for $5 \mathrm{mg} / \mathrm{kg}$ (Table 1). This may have been due to the increased number of animals in the $1 \mathrm{mg} / \mathrm{kg}$ group ( $n=7)$, as opposed to the $5 \mathrm{mg} / \mathrm{kg}$ group $(n=4)$. The administration of $5 \mathrm{mg} / \mathrm{kg}$ AR-C155858 did result in a decrease in ABEC and $t_{\mathrm{d}}$, but these findings were not statistically significant (Fig. 1A; Table 1). AR-C155858 did not have an impact on GHB toxicokinetics when administered as a $5 \mathrm{mg} / \mathrm{kg}$ intravenous bolus 60 minutes after the administration of GHB (Fig. 1B; Supplemental Table 1).

Impact of AZD3965 Pretreatment on Intravenous GHB TK/TD. When AZD3965 was administered 5 minutes prior to intravenous GHB there was a large reduction in the TD effects of GHB (Fig. 2A). All TD parameters were reduced in a statistically significant manner (Table 2). Two out of the four animals did not exhibit any sedation (ABEC and $t_{\mathrm{d}}$ of 0 , data not shown). The TK of GHB was also altered; there was a statistically significant reduction in AUC, which was a result of an increase in total and nonrenal clearance. There was no effect on $\mathrm{CL}_{\mathrm{R}}$ with AZD3965 pretreatment (Fig. 2B; Table 2).

Impact of AZD3965 on Intravenous GHB TK/TD. Administration of AZD3965 following intravenous GHB had

TABLE 1

Impact of post-treatment with AR-C155858 on intravenous GHB toxicodynamics

Data are presented as mean (S.D.). $n=4-7 .{ }^{*} P<0.05 ; * * P<0.01$ determined by one-way ANOVA with Dunnett's post hoc test for toxicodynamics and by Student's $t$ test for toxicokinetics.

\begin{tabular}{lccc}
\hline & & & $60 \mathrm{~min}$ PD \\
\cline { 3 - 4 } Toxicodynamic Parameter & GHB $(14.4 \mathrm{mmol} / \mathrm{kg})$ & GHB $(14.4 \mathrm{mmol} / \mathrm{kg})+\mathrm{AR}-\mathrm{C} 155858(1 \mathrm{mg} / \mathrm{kg})$ & $\mathrm{GHB}(14.4 \mathrm{mmol} / \mathrm{kg})+\mathrm{AR}-\mathrm{C} 155858(5 \mathrm{mg} / \mathrm{kg})$ \\
\hline ABEC (breaths) & $8.63 \times 10^{3}\left(2.64 \times 10^{3}\right)$ & $3.40 \times 10^{3}\left(1.83 \times 10^{3}\right)^{*}$ & $6.57 \times 10^{3}\left(2.30 \times 10^{3}\right)$ \\
$E_{\max }($ breaths $/ \mathrm{min})$ & $18.0(9.45)$ & $32.5(13.7)^{*}$ & $21.7(7.09)$ \\
$t_{\mathrm{d}}(\min )$ & $223(77.5)$ & $108(39.6)^{* *}$ & $161(36.4)$ \\
\hline
\end{tabular}

$E_{\max }$, maximum effect of GHB on breathing frequency; PD, postdose. 

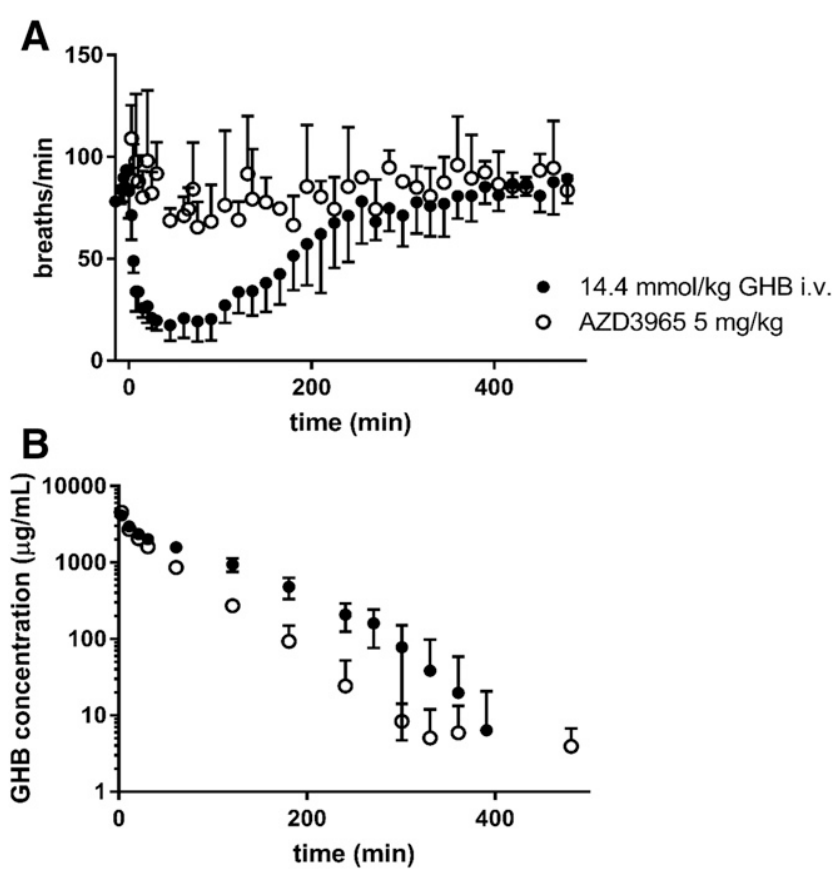

Fig. 2. Effect of AZD3965 pretreatment on intravenous GHB-induced respiratory depression (A) and GHB toxicokinetics (B). GHB was administered as a $14.4 \mathrm{mmol} / \mathrm{kg}$ intravenous bolus at time 0 . AZD3965 was administered 5 minutes prior to GHB as an intravenous bolus of $5 \mathrm{mg} / \mathrm{kg}$ (open circles). Data are presented as mean \pm S.D. Control data (closed circles) for breathing frequency were obtained from Morse and Morris (2013a); control data for GHB TK were obtained from Vijay et al. (2015). $n=3-5$.

minimal impact on GHB-induced respiratory depression (Fig. 3, A and B; Supplemental Table 2). There was no effect of AZD2965 when administered at a $1 \mathrm{mg} / \mathrm{kg}$ dose 60 minutes after GHB (data not shown). There was improvement in ABEC and $t_{\mathrm{d}}$ with $5 \mathrm{mg} / \mathrm{kg}$ AZD3965 administration 60 and $120 \mathrm{~min}-$ utes after GHB; however, these improvements did not reach statistical significance (Fig. 3, A and B; Supplemental Table 2). There were no statistically significant changes in GHB toxicokinetics with AZD3965 treatment 60 or 120 minutes after an intravenous bolus of GHB (Fig. 3, C and D; Supplemental Table 2).

Impact of AZD3965 on Oral GHB TK/TD. When AZD3965 was administered 60 minutes after an oral dose of GHB $(14.4 \mathrm{mmol} / \mathrm{kg})$ there was a statistically significant decrease in ABEC and $t_{\mathrm{d}}$ (Fig. 4A; Table 3). All animals were awake within 20 minutes of administration of AZD3965. There was an increase in breathing frequency with the administration of AZD3965 120 minutes after oral GHB, and a decrease in ABEC and $t_{\mathrm{d}}$; however, this was only significant for $t_{\mathrm{d}}$ (Fig. 4B; Table 3). This may be due to high variability in the control data. When AZD3965 was administered 60 minutes after oral $\mathrm{GHB}$, there was a statistically significant increase in $\mathrm{CL} / \mathrm{F}$ and $\mathrm{CL}_{\mathrm{R}}$, resulting in a corresponding decrease in AUC (Fig. 4C; Table 3). When the administration of AZD3965 was delayed 120 minutes after GHB administration, there was no effect on GHB TK (Fig. 4D; Table 3).

Impact of AZD3965 on Oral GBL TK/TD. AZD3965 reduced GHB-induced respiratory depression when administered after oral GBL (5.77 mmol/kg) (Fig. 5, A and B; Table 4). There was a statistically significant reduction in $t_{\mathrm{d}}$ when AZD3965 was administered 60 and 120 minutes after oral GBL (Table 4). When AZD3965 was administered 60 minutes after oral GBL, there was a reduction in ABEC as well (Table 4). AZD3965 was also effective at improving the TK of oral GBL, regardless of time of administration (Fig. 5, C and D; Table 4). When AZD3965 was administered 60 minutes after GBL, there was a significant reduction in AUC and a significant increase in $\mathrm{CL} / \mathrm{F}, \mathrm{CL}_{\mathrm{R}}$, and urinary excretion (Fig. 5C; Table 4); when administered 120 minutes after GBL, AUC was significantly decreased and $C L / F$ was significantly increased but $C_{R}$ was not significantly altered (Table 4).

Impact of AZD3965 on Brain-to-Plasma Partitioning of GHB Following Oral GHB Administration. AZD3965 did not have an effect on GHB brain-to-plasma partitioning 5 minutes after AZD3965 treatment (Fig. 6; Table 5). One hour after AZD3965 was administered, corresponding to 120 minutes after GHB administration, there was a large reduction in GHB brain concentrations for animals treated with AZD3965 compared with control animals (Fig. 6; Table 5). GHB concentrations for AZD3965-treated animals were not distinguishable from blank brain samples 1 hour after AZD3965 administration (data not shown).

\section{Discussion}

GHB has many approved pharmaceutical uses, including the treatment of narcolepsy and alcohol withdrawal; however, the use of this compound is limited by its abuse (Carter et al., 2009; White, 2017). GHB, and its prodrug GBL, are abused for their desirable effects such as euphoria; however, abuse of GHB carries the risk of hypothermia, coma, and respiratory

TABLE 2

Impact of pretreatment with AZD3965 on intravenous GHB toxicodynamics and toxicokinetics Data are presented as mean (S.D.). $n=3-5$. $* * P<0.01$; $* * * P<0.0001$ determined by Student's $t$ test.

\begin{tabular}{lcc}
\hline Parameter & GHB $(14.4 \mathrm{mmol} / \mathrm{kg})$ & $\begin{array}{c}\text { GHB }(14.4 \mathrm{mmol} / \mathrm{kg})+\mathrm{AZD} 3965 \\
(5 \mathrm{mg} / \mathrm{kg}) 5 \mathrm{~min} \text { Prior }\end{array}$ \\
\hline Toxicodynamics & & \\
ABEC (breaths) & $7.61 \times 10^{3}\left(2.27 \times 10^{3}\right)$ & $159(220)^{* * *}$ \\
$E_{\text {max }}($ breaths $/ \mathrm{min})$ & $18.0(2.95)$ & $59.3(8.61)^{* * *}$ \\
$t_{\mathrm{d}}(\mathrm{min})$ & $230(22.7)$ & $26(46)^{* *}$ \\
Toxicokinetics & & \\
AUC $(\mathrm{mg}$ min $/ \mathrm{ml})$ & $295(40.1)$ & $171(3.00)^{*}$ \\
$\mathrm{CL}_{\mathrm{T}}(\mathrm{ml} / \mathrm{min})$ & $5.15(0.706)$ & $8.77(0.155)^{* * *}$ \\
$\mathrm{CL}_{\mathrm{R}}(\mathrm{ml} / \mathrm{min})$ & $3.45(1.18)$ & $4.31(2.24)$ \\
$\mathrm{CL}_{\mathrm{NR}}(\mathrm{ml} / \mathrm{min})$ & $1.70(0.559)$ & $4.47(2.17)^{* *}$ \\
Urinary recovery $(\%)$ & $65.7(13.3)$ & $49.0(25.4)$ \\
\hline
\end{tabular}

$\mathrm{CL}_{\mathrm{NR}}$, nonrenal clearance; $\mathrm{CL}_{\mathrm{T}}$, total clearance; $E_{\max }$, maximum effect of GHB on breathing frequency. 
A
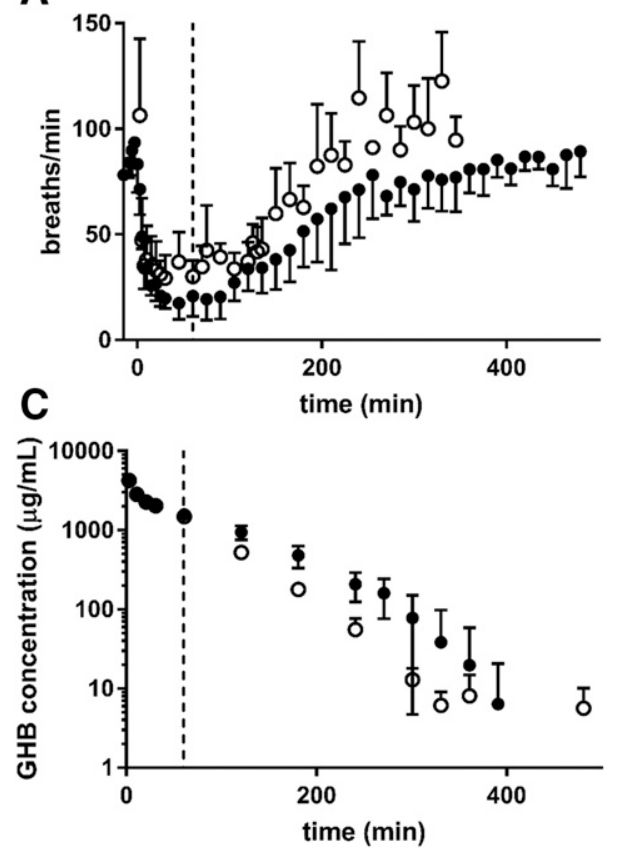

B
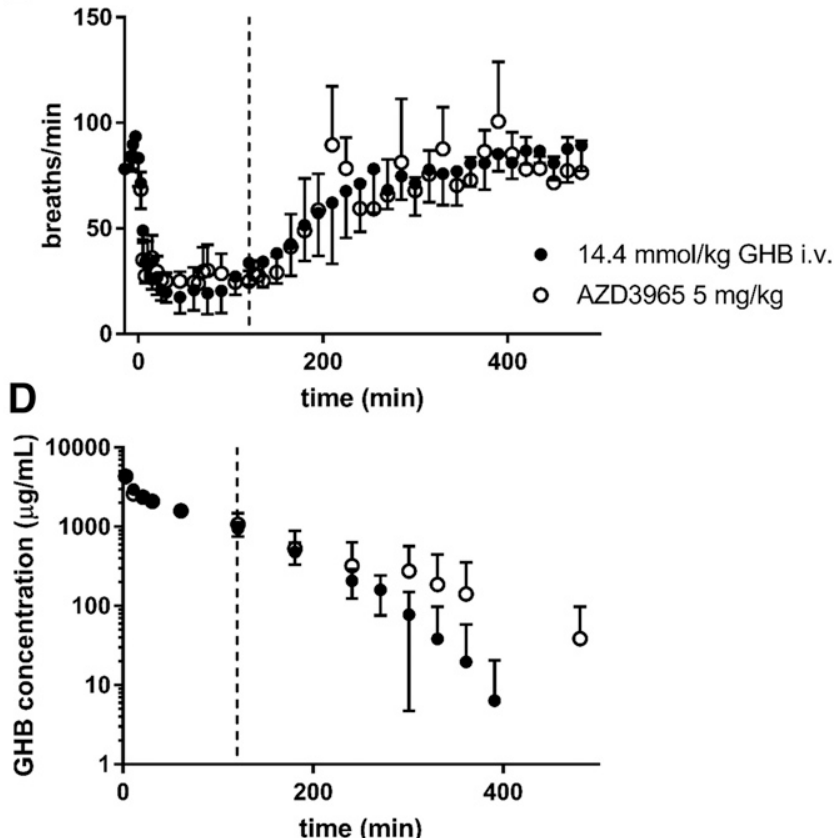

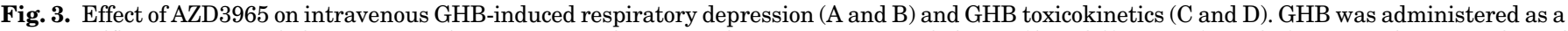

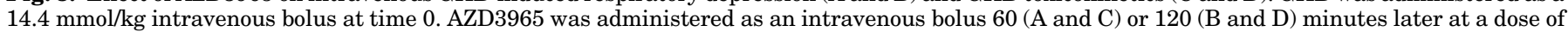

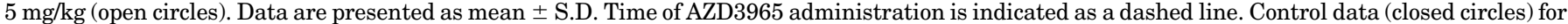
breathing frequency were obtained from Morse and Morris (2013a); control data for GHB TK were obtained from Vijay et al (2015). $n=4$ to 5 .

depression, which can be fatal (White, 2017). Recently, there has been a reported increase in GHB-related fatalities, heightening the need for a specific treatment of GHB/GBL overdose (Hockenhull et al., 2017). The involvement of MCT1, a proton-dependent transporter, at key sites for GHB TK and
TD makes this transporter an attractive target for GHB overdose treatment. MCT1 mediates the uptake of GHB to the brain, where GHB exerts its TD effects as a $\mathrm{GABA}_{\mathrm{B}}$ receptor agonist (Carai et al., 2001; Kaupmann et al., 2003; Goodwin et al., 2005, 2009). MCT1 also limits $\mathrm{CL}_{\mathrm{R}}$ of $\mathrm{GHB}$ by
A
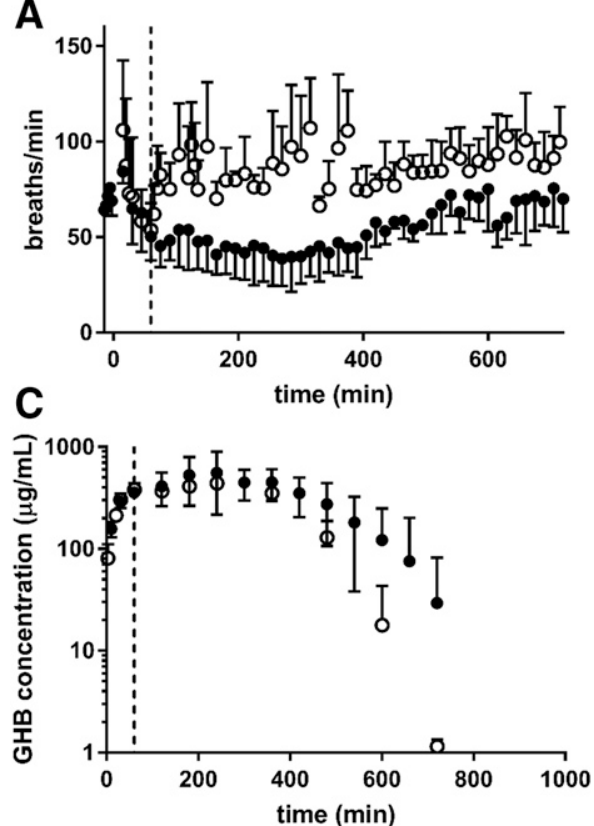

B
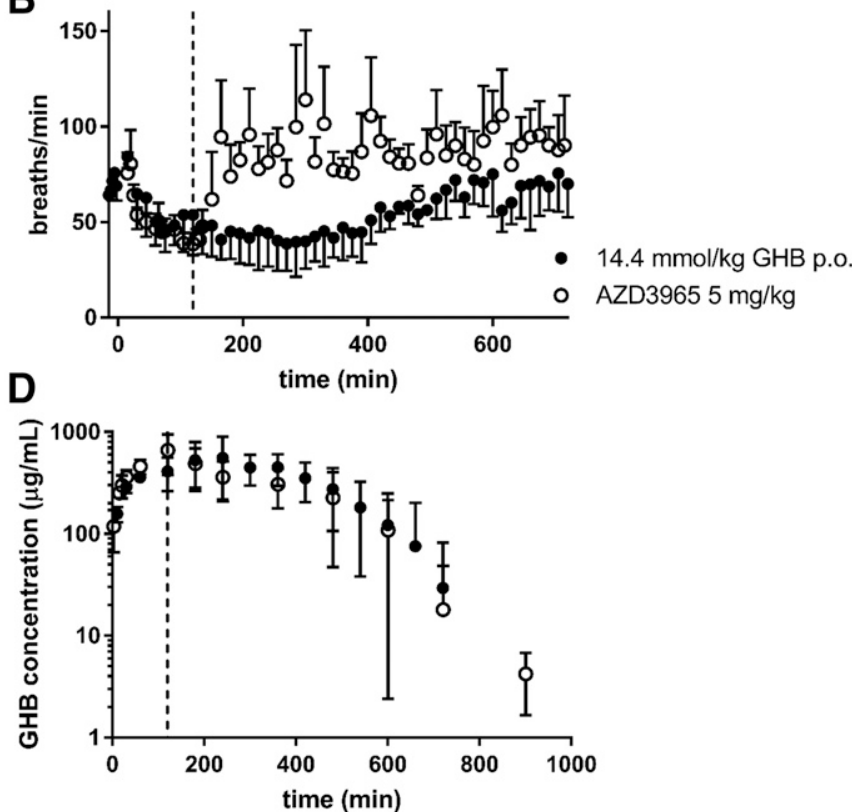

Fig. 4. Effect of AZD3965 on oral GHB breathing frequency (A and B) and GHB toxicokinetics (C and D). GHB (14.4 mmol/kg) was administered via oral gavage at time 0. AZD3965 was administered as an intravenous bolus 60 (A and C) or 120 (B and D) minutes later at a dose of $5 \mathrm{mg} / \mathrm{kg}$ (open circles). Data are presented as mean \pm S.D. Time of AZD3965 administration is indicated as a dashed line. Control data (closed circles) for breathing frequency were obtained from Morse and (Morris 2013a); control data for GHB TK were obtained from Vijay et al. (2015). $n=4-7$. 
TABLE 3

Impact of post-treatment with AZD3965 on oral GHB toxicodynamics and toxicokinetics

Data are presented as mean (S.D.). $n=4-7 .{ }^{*} P<0.05 ;{ }^{*} P<0.01$ determined by Student's $t$ test (for toxicodynamics) and one-way ANOVA with Dunnett's post hoc test (for toxicokinetics)

\begin{tabular}{|c|c|c|c|c|}
\hline \multirow{2}{*}{ Parameter } & \multicolumn{2}{|c|}{ GHB $(14.4 \mathrm{mmol} / \mathrm{kg})$} & \multicolumn{2}{|c|}{ GHB $(14.4 \mathrm{mmol} / \mathrm{kg})+$ AZD3965 $(5 \mathrm{mg} / \mathrm{kg})$} \\
\hline & $60 \mathrm{~min}$ & $120 \mathrm{~min}$ & $60 \min \mathrm{PD}$ & $120 \min$ PD \\
\hline \multicolumn{5}{|l|}{ Toxicodynamics } \\
\hline$t_{\mathrm{d}}(\mathrm{h})$ & $341(214)$ & $300(180)$ & $71.4(32.5)^{*}$ & $64.9(54.3)^{*}$ \\
\hline \multicolumn{5}{|l|}{ Toxicokinetics } \\
\hline AUC $(\mathrm{mg} * \mathrm{~min} / \mathrm{ml})$ & $234(31.1)$ & & $171(18.4)^{*}$ & $216(59.3)$ \\
\hline Urinary recovery $(\%)$ & $28.1(10.2)$ & & $36.8(4.10)$ & $34.4(9.71)$ \\
\hline$C_{\max }(\mu \mathrm{g} / \mathrm{ml})$ & $659(259)$ & & $458(54.8)$ & $720(165)$ \\
\hline$t_{\max }(\mathrm{h})$ & $309(140)$ & & $166(75.5)$ & $193(161)$ \\
\hline
\end{tabular}

$\mathrm{CL}_{\mathrm{NR}}$, nonrenal clearance; $\mathrm{CL}_{\mathrm{NR}} / \mathrm{F}$, oral nonrenal clearance; $E_{\max }$, maximum effect of $\mathrm{GHB}$ on breathing frequency; PD, postdose; $t_{\mathrm{max}}$, time of maximum concentration.

facilitating the reabsorption of the compound after it is filtered through the glomerulus and enters the proximal tubule (Morris et al., 2005). Previous studies in our laboratory have indicated that pretreatment, or treatment immediately following GHB administration, with MCT1 inhibitors can prevent GHB-induced respiratory depression (Vijay et al., 2015). Early treatment with MCT1 inhibitors led to an increase in GHB $\mathrm{CL}_{\mathrm{R}}$ in preclinical and clinical studies (Morris et al., 2011; Vijay et al., 2015). The aim of this work was to further investigate the utility and mechanism of MCT1 inhibition for the treatment of GHB/GBL overdose.

AR-C155858 and AZD3965 are potent and partially selective MCT1 inhibitors. AR-C155858 was developed in a series of compounds designed to be immunosuppressants by targeting T-effector cells and sparing T-regulatory cells through modulation by MCT1 (Påhlman et al., 2013). AR-C155858 has a high affinity for MCT1 with a $K_{\mathrm{i}}$ value of only $2.3 \mathrm{nM}$ (Ovens et al., 2010). AZD3965 is also partially selective for MCT1 and is 6-fold more selective than for monocarboxylate transporter 2 , which has a $K_{\mathrm{i}}$ value of $1.6 \mathrm{nM}$ (Bola et al., 2014). AZD3965 has been in phase I clinical trials for treatment of advanced solid tumors (Bola et al., 2014). Due to their potency and selectivity, these two MCT1 inhibitors were used in this study.

Administration of AR-C155858 5 minutes after intravenous GHB administration has been shown previously to reduce GHB-induced respiratory depression and limit GHB exposure
A
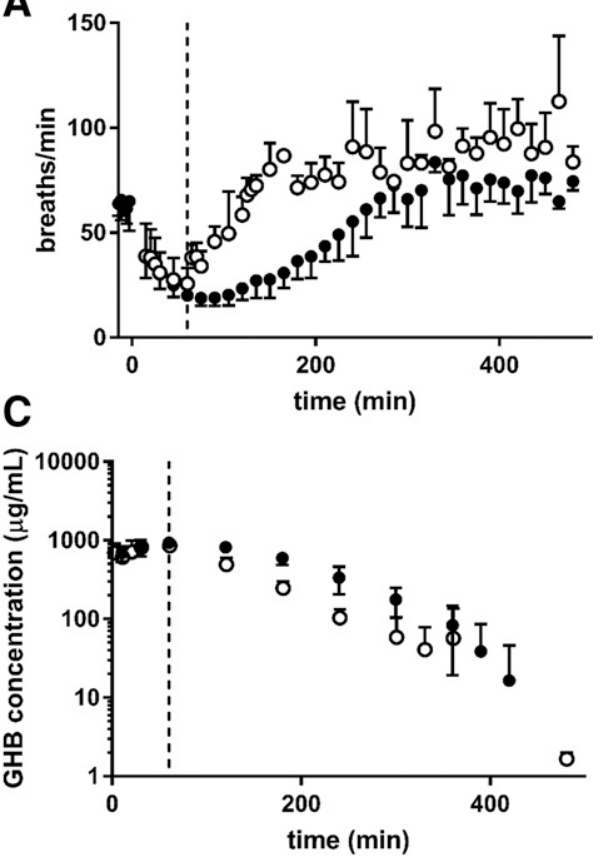

B
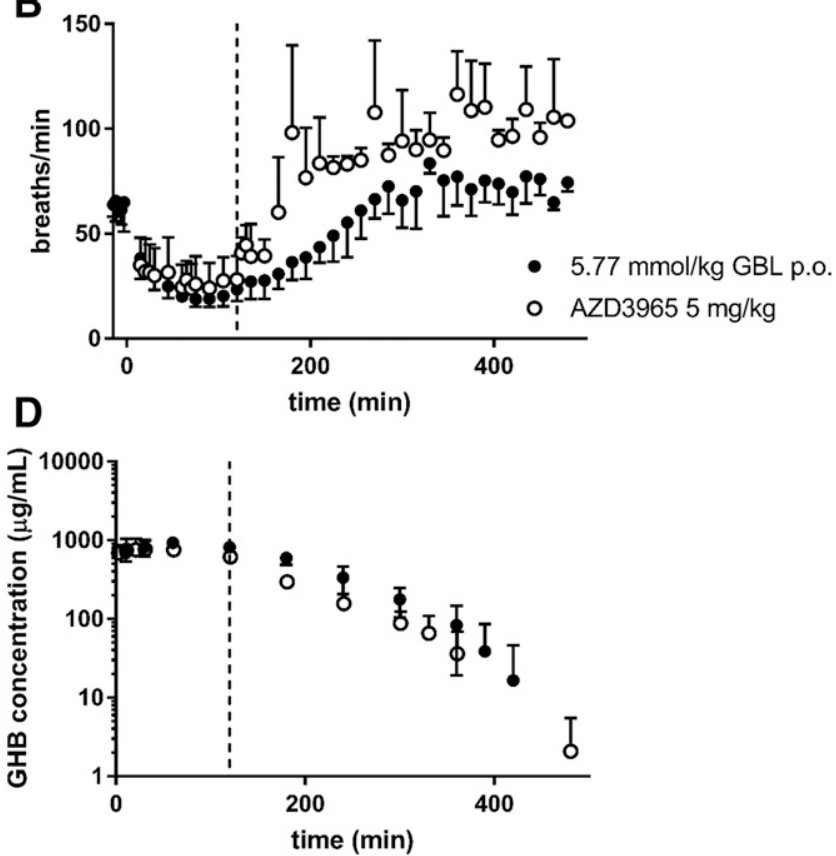

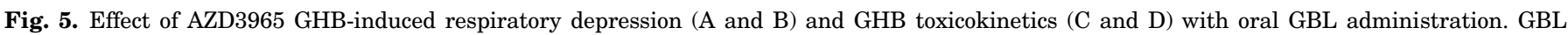

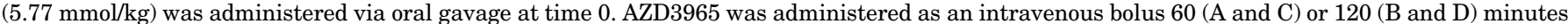

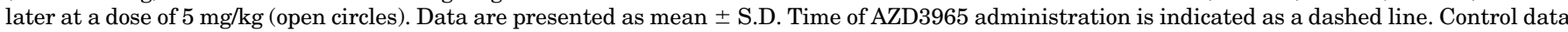

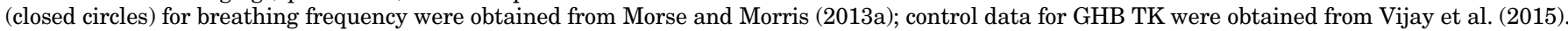
$n=4$ to 5 . 
TABLE 4

Impact of post-treatment with AZD3965 on GHB toxicodynamics and toxicokinetics with oral GBL administration Data are presented as mean (S.D.). $n=4$ to 5 . ${ }^{*} P<0.05$; ${ }^{*} P<0.01$; ${ }^{* * *} P<0.001$ determined by Student's $t$ test (for toxicodynamics) and one-way ANOVA with Dunnett's post hoc test (for toxicokinetics).

\begin{tabular}{|c|c|c|c|c|}
\hline \multirow{2}{*}{ Parameter } & \multicolumn{2}{|c|}{ GBL $(5.77 \mathrm{mmol} / \mathrm{kg})$} & \multicolumn{2}{|c|}{ GBL $(5.77 \mathrm{mmol} / \mathrm{kg})+$ AZD3965 $(5 \mathrm{mg} / \mathrm{kg})$} \\
\hline & $60 \mathrm{~min}$ & $120 \mathrm{~min}$ & $60 \min \mathrm{PD}$ & $120 \mathrm{~min}$ PD \\
\hline \multicolumn{5}{|l|}{ Toxicodynamics } \\
\hline ABEC (breaths) & $6.02 \times 10^{3}\left(2.06 \times 10^{3}\right)$ & $3.42 \times 10^{3}\left(1.62 \times 10^{3}\right)$ & $1.74 \times 10^{3}(709)^{* *}$ & $1.55 \times 10^{3}(704)$ \\
\hline$E_{\max }($ breaths/min) & $18.0(2.95)$ & $23.4(5.61)$ & $25.3(6.85)$ & $27.1(9.80)$ \\
\hline$t_{\mathrm{d}}(\mathrm{h})$ & $185(22.7)$ & $125(22.7)$ & $66.8(20.6)^{* * *}$ & $54.3(16.7)^{* *}$ \\
\hline \multicolumn{5}{|l|}{ Toxicokinetics } \\
\hline AUC $(\mathrm{mg} * \min / \mathrm{ml})$ & $194(17.4)$ & & $131(22.6)^{* * *}$ & $141(9.78) * *$ \\
\hline $\mathrm{CL} / \mathrm{F}(\mathrm{ml} / \mathrm{min} * \mathrm{~kg})$ & $2.58(0.216)$ & & $3.87(0.636)^{* *}$ & $3.54(0.250)^{* *}$ \\
\hline $\mathrm{CL}_{\mathrm{R}}(\mathrm{ml} / \mathrm{min} * \mathrm{~kg})$ & $0.568(0.254)$ & & $1.45(0.219)^{* *}$ & $1.00(0.274)$ \\
\hline $\mathrm{CL}_{\mathrm{NR}} / \mathrm{F}(\mathrm{ml} / \mathrm{min} * \mathrm{~kg})$ & $2.01(0.149)$ & & $2.42(0.560)$ & $2.54(0.391)$ \\
\hline Urinary recovery $(\%)$ & $21.6(8.59)$ & & $37.7(2.02)^{*}$ & $28.5(8.06)$ \\
\hline$C_{\max }(\mu \mathrm{g} / \mathrm{ml})$ & $972(93.3)$ & & $904(107)$ & $925(186)$ \\
\hline$t_{\max }(\mathrm{h})$ & $67.5(37.7)$ & & $36.0(17.3)$ & $27(20.7)$ \\
\hline
\end{tabular}

$\mathrm{CL}_{\mathrm{NR}}$, nonrenal clearance; $\mathrm{CL}_{\mathrm{NR}} / \mathrm{F}$, oral nonrenal clearance; $E_{\mathrm{max}}$, maximum effect of $\mathrm{GHB}$ on breathing frequency; $\mathrm{PD}$, postdose; $t_{\mathrm{max}}$, time of maximum concentration.

through an increase in $\mathrm{CL}_{\mathrm{R}}$ (Vijay et al., 2015). In this study, when AR-C155858 administration was delayed 60 minutes after GHB, there was a reduction in ABEC for breathing frequency but there was no effect on GHB TK. Results with another MCT1 inhibitor, AZD3965, were similar; there was a reduction in TD for GHB but not for TK when treatment was delayed 60 minutes. Neither compound had an effect on GHB TK/TD when treatment was delayed 120 minutes after intravenous GHB administration.

While the success of treatment of GHB overdose following intravenous administration of the compound was dependent on the timing of administration of MCT1 inhibitors, it was important to assess the utility of these compounds after oral GHB and GBL administration. GHB and GBL are typically abused orally, and this route of administration is more relevant to a real world scenario. AZD3965 was successful in reducing the duration of effect for oral GHB TD regardless of time of administration. There was only a reduction in GHB TK when AZD3965 was administered 60 minutes after oral GHB. Similar results were obtained with AZD3965 following oral GBL administration; at both times of administration the

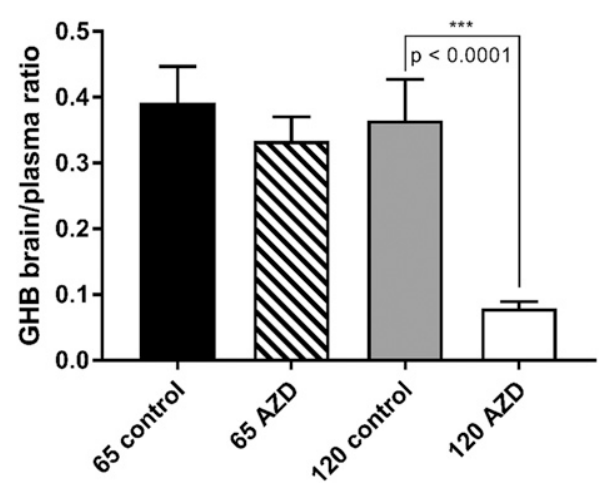

Fig. 6. Effect of AZD3965 on GHB brain-to-plasma partitioning with oral GHB administration. GHB $(14.4 \mathrm{mmol} / \mathrm{kg})$ was administered via oral gavage at time 0 . Control groups were sacrificed at 65 and 120 minutes [65 control (black bar) and 120 control (gray bar), respectively]. Treatment groups were administered intravenous AZD3965 60 minutes after GHB administration. Treatment groups were sacrificed at 65 and 120 minutes [65 AZD (striped bar) and 120 AZD (clear bar), respectively]. Whole brain concentrations were compared with plasma concentrations. Bars represent mean data, error bars are + S.D. $n=4$. compound reduced the TD effects of GHB. AZD3965 was more effective at reducing GHB TK after oral GBL administration as opposed to oral GHB administration. $\mathrm{CL} / \mathrm{F}$ was increased with oral GBL when treatment was delayed 60 and 120 minutes.

Our results indicated that GHB TD effects could not be predicted by GHB plasma exposure. Therefore, to understand how AZD3965 was acting as an effective treatment of GHB/GBL overdose without dramatically increasing $\mathrm{CL}_{\mathrm{R}}$ across all treatment groups, as expected, the brain concentrations of GHB were assessed. When AZD3965 was administered 60 minutes after oral GHB, there was pronounced reduction in the GHB brain-to-plasma ratio. This reduction was not immediate, since there was no change in the GHB brain-to-plasma ratio 5 minutes after AZD3965 administration (65 minutes after GHB administration). However, 60 minutes after AZD3965 treatment (120 minutes after GHB administration) the control group had a brain-to-plasma ratio over 4-fold higher than the AZD3965-treated animals. This indicates that the reduction in GHB TD with MCT1 inhibition with AZD3965 is driven by changes in brain concentrations of GHB, not plasma concentrations. While AZD3965 is effective at reducing GHB TK in some treatment groups, the main driver for efficacy of this compound against GHB/GBL overdose is mediated through its reduction of GHB brain exposure. The reduction in the GHB brain-to-plasma ratio is mediated by the impact of AZD3965 on MCT1 transport at the BBB. While the mechanism of inhibition for AZD3965 at the BBB has not been characterized, the reduction of the GHB brain-to-plasma

\section{TABLE 5}

Impact of AZD3965 on GHB brain-to-plasma partitioning with oral GHB administration

Data are presented as mean (S.D.). $n=4 .{ }^{* * *} P<0.01$ determined by Student's $t$ test.

\begin{tabular}{lcc}
\hline \multirow{2}{*}{$\begin{array}{c}\text { Time of Sacrifice } \\
\text { after GHB } \\
\text { Administration }\end{array}$} & $\begin{array}{c}\text { Oral GHB } \\
(14.4 \mathrm{mmol} / \mathrm{kg})\end{array}$ & $\begin{array}{r}\text { Oral GHB }(14.4 \mathrm{mmol} / \mathrm{kg})+\text { AZD3965 } \\
(5 \mathrm{mg} / \mathrm{kg}) 60 \mathrm{~min} \text { PD }\end{array}$ \\
\cline { 2 - 3 } \multicolumn{1}{c}{$\min$} & & \\
65 & $0.365(0.0627)$ & $0.334(0.365)$ \\
120 & $0.392(0.0551)$ & $0.0789(0.0104) * * *$ \\
\hline
\end{tabular}

PD, postdose. 
ratio after administration of AZD3965 indicates the uptake of GHB is inhibited at this site. Further studies investigating the mechanism of inhibition for AZD3965 at the BBB are required to confirm this finding. Based on the results obtained in this study, AZD3965 is expected to be a safe and effective treatment of $\mathrm{GHB} / \mathrm{GBL}$ overdose.

\section{Acknowledgments}

The authors acknowledge Vivian Rodriguez-Cruz for assistance during the in vivo experiments.

\section{Authorship Contributions}

Participated in research design: Follman, Morris.

Conducted experiments: Follman.

Performed data analysis: Follman, Morris.

Wrote or contributed to the writing of the manuscript: Follman, Morris.

\section{References}

Bola BM, Chadwick AL, Michopoulos F, Blount KG, Telfer BA, Williams KJ, Smith PD, Critchlow SE, and Stratford IJ (2014) Inhibition of monocarboxylate transporter-1 (MCT1) by AZD3965 enhances radiosensitivity by reducing lactate transport. Mol Cancer Ther 13:2805-2816.

Carai MA, Colombo G, Brunetti G, Melis S, Serra S, Vacca G, Mastinu S, Pistuddi AM, Solinas C, Cignarella G, et al. (2001) Role of $\mathrm{GABA}_{\mathrm{B}}$ receptors in the sedative/hypnotic effect of $\gamma$-hydroxybutyric acid. Eur J Pharmacol 428:315-321.

Carter LP, Pardi D, Gorsline J, and Griffiths RR (2009) Illicit gammahydroxybutyrate (GHB) and pharmaceutical sodium oxybate (Xyrem): differences in characteristics and misuse. Drug Alcohol Depend 104:1-10.

Felmlee MA, Roiko SA, Morse BL, and Morris ME (2010) Concentration-effect relationships for the drug of abuse $\gamma$-hydroxybutyric acid. J Pharmacol Exp Ther 333:764-771.

Giarman NJ and Roth RH (1964) Differential estimation of gamma-butyrolactone and gamma-hydroxybutyric acid in rat blood and brain. Science 145:583-584.

Goodwin AK, Brown PR, Jansen EE, Jakobs C, Gibson KM, and Weerts EM (2009) Behavioral effects and pharmacokinetics of gamma-hydroxybutyrate (GHB) precursors gamma-butyrolactone (GBL) and 1,4-butanediol (1,4-BD) in baboons. Psychopharmacology (Berl) 204:465-476.

Goodwin AK, Froestl W, and Weerts EM (2005) Involvement of gammahydroxybutyrate (GHB) and GABA-B receptors in the acute behavioral effects of GHB in baboons. Psychopharmacology (Berl) 180:342-351.

Hockenhull J, Murphy KG, and Paterson S (2017) An observed rise in $\gamma$-hydroxybutyrate-associated deaths in London: evidence to suggest a possible link with concomitant rise in chemsex. Forensic Sci Int 270:93-97.

Jensen K and Mody I (2001) GHB depresses fast excitatory and inhibitory synaptic transmission via $\mathrm{GABA}_{\mathrm{B}}$ receptors in mouse neocortical neurons. Cereb Cortex 11 $424-429$
Kaupmann K, Cryan JF, Wellendorph P, Mombereau C, Sansig G, Klebs K, Schmutz M, Froestl W, van der Putten $\mathrm{H}$, Mosbacher J, et al. (2003) Specific $\gamma$-hydroxybutyrate-binding sites but loss of pharmacological effects of $\gamma$-hydroxybutyrate in

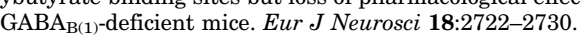

Lettieri J and Fung HL (1976) Absorption and first-pass metabolism of 14C-gammahydroxybutyric acid. Res Commun Chem Pathol Pharmacol 13:425-437.

Marinetti LJ, Isenschmid DS, Hepler BR, and Kanluen S (2005) Analysis of GHB and 4-methyl-GHB in postmortem matrices after long-term storage. $J$ Anal Toxicol 29: 41-47.

Morris ME and Felmlee MA (2008) Overview of the proton-coupled MCT (SLC16A) family of transporters: characterization, function and role in the transport of the drug of abuse gamma-hydroxybutyric acid. AAPS $J$ 10:311-321.

Morris ME, Hu K, and Wang Q (2005) Renal clearance of $\gamma$-hydroxybutyric acid in rats: increasing renal elimination as a detoxification strategy. J Pharmacol Exp Ther 313:1194-1202.

Morris ME, Morse BL, Baciewicz GJ, Tessena MM, Acquisto NM, Hutchinson DJ, and Dicenzo R (2011) Monocarboxylate transporter inhibition with osmotic diuresis increases $\gamma$-hydroxybutyrate renal elimination in humans: a proof-of-concept study. J Clin Toxicol 1:1000105.

Morse BL and Morris ME (2013a) Effects of monocarboxylate transporter inhibition on the oral toxicokinetics/toxicodynamics of $\gamma$-hydroxybutyrate and $\gamma$-butyrolactone. J Pharmacol Exp Ther 345:102-110

Morse BL and Morris ME (2013b) Toxicokinetics/toxicodynamics of $\gamma$-hydroxybutyrate-ethanol intoxication: evaluation of potential treatment strategies. $J$ Pharmacol Exp Ther 346:504-513.

Morse BL, Vijay N, and Morris ME (2012) $\gamma$-Hydroxybutyrate (GHB)-induced respiratory depression: combined receptor-transporter inhibition therapy for treatment in GHB overdose. Mol Pharmacol 82:226-235.

Ovens MJ, Davies AJ, Wilson MC, Murray CM, and Halestrap AP (2010) ARC155858 is a potent inhibitor of monocarboxylate transporters MCT1 and MCT2 that binds to an intracellular site involving transmembrane helices 7-10. Biochem $J$ 425:523-530.

Påhlman C, Qi Z, Murray CM, Ferguson D, Bundick RV, Donald DK, and Ekberg H (2013) Immunosuppressive properties of a series of novel inhibitors of the monocarboxylate transporter MCT-1. Transpl Int 26:22-29.

Vijay N and Morris ME (2014) Role of monocarboxylate transporters in drug delivery to the brain. Curr Pharm Des 20:1487-1498.

Vijay N, Morse BL, and Morris ME (2015) A novel monocarboxylate transporter inhibitor as a potential treatment strategy for $\gamma$-hydroxybutyric acid overdose. Pharm Res 32:1894-1906.

White CM (2017) Pharmacologic, pharmacokinetic, and clinical assessment of illicitly used $\gamma$-hydroxybutyrate. J Clin Pharmacol 57:33-39.

Zhang Y, Huo M, Zhou J, and Xie S (2010) PKSolver: an add-in program for pharmacokinetic and pharmacodynamic data analysis in Microsoft Excel. Comput Methods Programs Biomed 99:306-314.

Address correspondence to: Dr. Marilyn E. Morris, Department of Pharmaceutical Sciences, School of Pharmacy and Pharmaceutical Sciences, University at Buffalo, The State University of New York, Buffalo, NY 14214. E-mail: memorris@buffalo.edu 